\title{
Three-dimensional finite element modeling from CT images of tooth and its validation
}

Kiyoshi TAJIMA ${ }^{1}$, Ker-Kong CHEN $^{2}$, Nobusuke TAKAHASHI ${ }^{1}$, Naoaki NODA ${ }^{3}$, Yuki NAGAMATSU ${ }^{1}$ and Hiroshi KAKIGAWA ${ }^{1}$

\author{
${ }^{1}$ Division of Biomaterials, Kyushu Dental College, 2-6-1 Manazuru, Kokurakita, Kitakyushu 803-8580, Japan \\ ${ }^{2}$ Division of Operative Dentistry, Kyushu Dental College, 2-6-1 Manazuru, Kokurakita, Kitakyushu 803-8580, Japan \\ ${ }^{3}$ Mechanical Engineering Department, Kyushu Institute of Technology, 1-1 Sensui, Tobata, Kitakyushu 804-8550, Japan \\ Corresponding author, Kiyoshi TAJIMA; E-mail: tajima@kyu-dent.ac.jp
}

\begin{abstract}
The aim of this study was to develop a three-dimensional (3D) finite element (FE) model of a sound extracted human second premolar from micro-CT data using commercially available software tools. A detailed 3D FE model of the tooth could be constructed and was experimentally validated by comparing strains calculated in the FE model with strain gauge measurement of the tooth under loading. The regression coefficient and its standard error in the regression analysis between strains calculated by the FE model and measured with strain gauge measurement were 0.82 and 0.06 , respectively, and the correlation coefficient was found to be highly significant. These results suggested that an FE model reconstructed from micro-CT data could be used as a valid model to estimate the actual strains with acceptable accuracy.
\end{abstract}

Key words: Finite element modeling, Tooth, CT

Received Sep 5, 2008: Accepted Oct 23, 2008

\section{INTRODUCTION}

Finite element (FE) analysis was initially implemented in dentistry in the early 1970s and has been increasingly used to analyze biomechanical behaviors of dental materials and biological structures $^{1-3)}$. In FE analysis, it is necessary to construct an FE model of the structures to be examined. An FE model with a topological description of geometrical and structural asymmetry can be modeled in either a two-dimensional (2D) or three-dimensional (3D) approach ${ }^{4-6)}$. Recently, 3D models for use in $\mathrm{FE}$ analysis have became popular in the study of dental biomechanics, because they allow better understanding of the mechanical and fracture behavior of the dental tissues and structures, providing more realistic and accurate results resembling the actual occurrence in clinics ${ }^{6)}$ than do the simplified 2D models.

Accurate geometry is one of the important keys to successful 3D modeling of both sound and restored teeth in FE analysis. For complicated features of teeth, there were two previous methods of manual description: 1) use of standard anatomical data in the literature, and 2) use of cross-sectional histological images of teeth ${ }^{7,8)}$. These conventional ways to describe the 3D models often necessitated timeconsuming and labor-intensive effort, and resulted in a generally glossy simplification of the model geometry. Recently, an image acquisition method using computed topography (CT) has been extensively applied as a scanning tool for 3D-digitizing purposes in modeling of teeth ${ }^{9-15)}$, providing higher accuracy, faster digitization and finer description. In addition, several pioneering modeling techniques for CT data have been originally developed as in-house programs to extract the model geometry, because this method requires the extraction of inner and outer contours from CT data of teeth ${ }^{7-10)}$. More recently, several computer-aided techniques to extract the geometry have become commercially available with the industrial development of 3D image processing and reverse engineering techniques, facilitating the construction of FE models ${ }^{11-15)}$.

Validation of the FE model, one of the important problems in $\mathrm{FE}$ analysis ${ }^{17,18}$, has been previously reported by experimental verification through comparison of the results with those of laboratory or clinical tests of the teeth ${ }^{18-25}$. It is possible to compare the strain calculated in FE models and experimentally measured results using strain gauges, although the process is very difficult and tedious. Some researchers have previously indicated that $3 \mathrm{D}$ FE models reconstructed from cross-sectional histological images showed good agreement with experimental methods ${ }^{21-24)}$. However, until now, the resulting 3D FE models constructed from CT image data of the teeth have not been fully validated ${ }^{18,25)}$.

The purpose of this study was to develop a 3D FE model via a $\mathrm{CAD}$ model reconstructed from $\mathrm{CT}$ data of the extracted tooth, using commercially available software tools. Furthermore, the accuracy of the created FE model was experimentally validated by comparing strains calculated from the FE model 
with strain gauge measurements of the tooth under loading.

\section{MATERIALS AND METHODS}

\section{Tooth FE modeling from CT data}

The geometrical model was obtained by $3 \mathrm{D}$ reconstruction from CT images of a sound extracted human maxillary second premolar. The process required to reconstruct the $3 \mathrm{D} \mathrm{FE}$ model of the tooth was comprised of the following steps: 1) image acquisition, 2) geometry extraction, 3) surface modeling, 4) solid modeling, and 5) FE meshing.

For image acquisition, the tooth was scanned with an industrial micro-focus 3D X-ray system (HMX225-ACTIS+3, TESCO, Tokyo, Japan). The scan conditions were as follows: tube voltage, $131 \mathrm{kV}$; exposure dose, $57 \mu \mathrm{A}$; image matrix, 512 x 512; geometric magnification, 14.3; slice thickness, 50 $\mu \mathrm{m}$. The obtained CT data were converted into a series of CT slice images with 8-bit tagged image file (TIF) format. The CT slice images were visualized with a free 3D DICOM viewer (INTAGE Realia, KGT, Tokyo, Japan).

For geometry extraction, the grayscale slice images were imported into a light-weight tool for the generation of $3 \mathrm{D}$ point clouds from the multi-slice images (Pilamaster, ISID, Tokyo, Japan). Three point clouds of the contours of the outer tooth and dentin region, and the boundary of the dentine and pulp were extracted from the CT slices. The cementum layer was included in the dentin portion of the tooth because of its thinness. Enamel was created in the following solid modeling. Each region was manually determined according to their different threshold values for a given grayscale image. Then, for the multi-slices, threshold-based segmentation and 3D stacking of the point clouds were automatically performed, resulting in the separate generation of $3 \mathrm{D}$ point clouds for the three regions. The respective point cloud data were exported in initial graphics exchange specification (IGES) format.

These points were then imported into an application tool for 3D reverse engineering and surface reconstruction (LeiosMesh, Datadesign, Nagoya, Japan) to generate the surface models. The input points were filtered to reduce their number to a level in which the accuracy of the models could be maintained, followed by triangular mesh creation, mesh healing by smoothing, and triangular mesh decimation. The finally obtained triangle mesh models were then fit with a stitched set of untrimmed nonuniform rational B-spline (NURBS) surfaces, resulting in surface models. The operations with this tool were performed completely automatically by clicking command menus.

Surface models of three regions were imported in
IGES format into a 3D CAD package (Rhinoceros, Robert McNeel \& Assoc., WA, USA) for manual creation of the respective volumetric (solid) models of tooth, dentin and pulp. After creating these solid models, a solid model of the enamel was created using Boolean operations ${ }^{26}$ for the tooth and dentin solid models. Finally, to assemble a solid model of the tooth consisting of enamel, dentin and pulp, the dentin model was added to the enamel model, while the pulp model was subtracted from the dentin model using Boolean operations. After assembly, unsuitable geometric features such as overlapping surfaces and small surfaces were manually corrected. The resulting assembled solid model of the tooth was finally exported in IGES format into a finite element pre- and post-processor (HyperMesh, Altair Engineering, MI, USA) to reconstruct an FE mesh model with a 3D 10-node tetrahedral structural solid element. This element is defined by 4 nodes at the corners of a triangular gimlet and 6 midside nodes. The meshing program caused some errors in meshing due to the unsuitable geometric features, which were manually corrected.

\section{Strain measurement with strain gauge technique}

Five sound extracted second premolars with similar dimensions were selected. The teeth were stored in 1.0\% chloramines to prevent dehydration. The actual strains of the extracted tooth under compressive loading with conic-type indentation were measured using the strain gauge technique ${ }^{27)}$ to verify the FE model. Figure 1 shows a diagram of the locations of strain gauges and loading points of the tooth. A uniaxial strain gauge (KFG-1N-120-C111 L1M2R, Kyowa, Tokyo, Japan) with a gauge length of $1 \mathrm{~mm}$ was bonded to the surface of the teeth. The bonded locations of strain gauge were four regions; the bucco-lingual cervical third regions of the crown and root while placing them below and above the cementoenamel junction (CEJ). The strain was measured in only one direction along the long axis of the tooth. Stainless rings $(30 \mathrm{~mm}$ in diameter and $26 \mathrm{~mm}$ in height) were filled with die stone, and the tooth was embedded to a level $2 \mathrm{~mm}$ apical to the CEJ, without covering the strain gauges as shown in Fig. 2. Following the embedding of the tooth, gauge leads were attached to a dynamic strain amplifier (DPM-711B, Kyowa, Tokyo, Japan).

A compressive load of $9 \mathrm{~kg}$ was applied to the loading points of the embedded tooth, as shown in Fig. 1, with an apparatus modified to apply a constant load. Loading points were as follows: buccal cusp (BC), the outer incline of the buccal cusp (BO), the inner incline of the buccal cusp (BI), lingual cusp (LC), the outer incline of the lingual cusp (LO) and the inner incline of the lingual cusp (LI). For $\mathrm{BC}$ and LC loading, the load was applied along the 

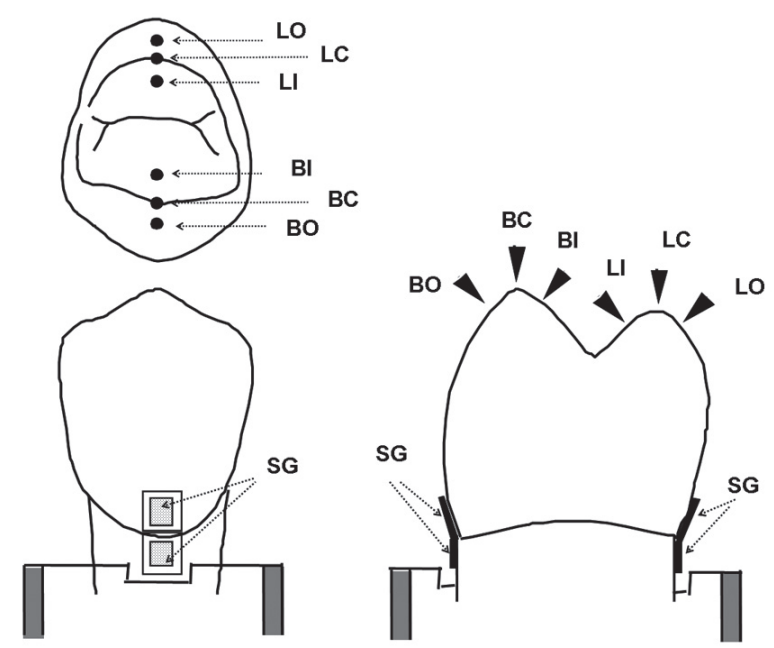

Fig. 1 Diagrams of four strain gauges bonded to the tooth and six occlusal points loaded.

$\mathrm{BC}$, buccal cusp; BO, the outer incline of the buccal cusp; BI, the inner incline of the buccal cusp; LC, lingual cusp; LO, the outer incline of the lingual cusp; LI, the inner incline of the lingual cusp; SG, strain gauges.

tooth axis bucco-lingually in the sagittal plane of the tooth. For the other loading points, the embedded tooth was secured in the apparatus so that it was aligned bucco-lingually at an angle of $-45^{\circ}$ or $45^{\circ}$ to the tooth axis for application with a load of $9 \mathrm{~kg}$.

\section{Strain calculation with the FE model}

The obtained FE mesh model was exported with the application program interface of HyperMesh to a general purpose FE program for engineering analysis (ANSYS Academic Teaching Introductory, CYBERNET, Tokyo, Japan). In the FE model, boundary conditions were applied to represent the conditions of experimental measurement with a strain gauge. The nodes on the root surface corresponding to the portion embedded with die stone in the strain gauge technique were constrained in the $\mathrm{x}, \mathrm{y}$, and $\mathrm{z}$ directions. A load of $88.26 \mathrm{~N}$ (9 kg) was applied to the nodes according to the loading points of the tooth in the strain gauge technique ${ }^{26)}$. Material properties of enamel and dentin (Young's modulus, Poisson's ratio) were as follows: enamel $60.6 \mathrm{GPa}, 0.30$; dentin: $18.3 \mathrm{GPa}, 0.30^{28)}$, and all materials were assumed to be homogeneous, isotropic and linear elastic. The pulp was modeled as void inside the dentin because the Young's modulus of pulp is negligibly small in comparison to those of enamel and dentin. Calculation for the FE model was performed with a workstation computer (Xeon $3.8 \mathrm{GHz}$ processor, 3.25 GB RAM, running Microsoft Windows XP Professional).

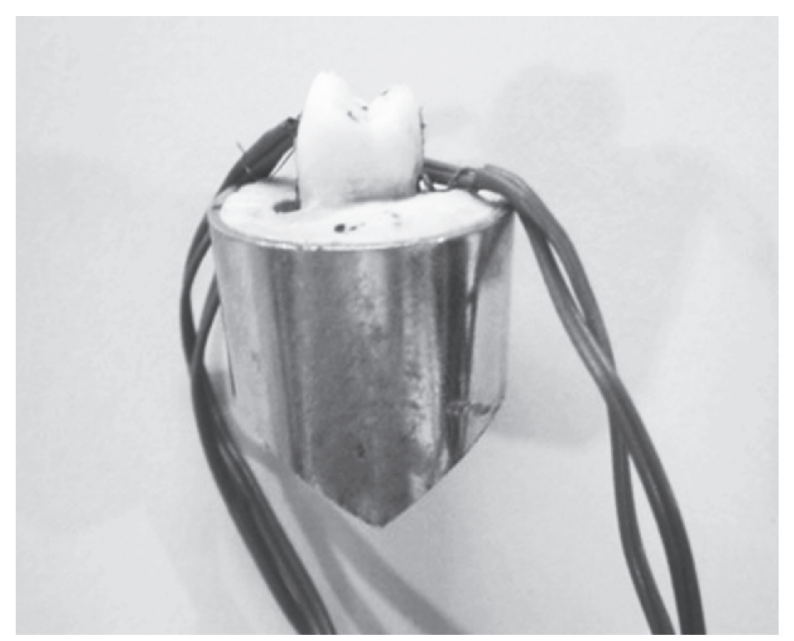

Fig. 2 Tooth-bonded strain gauges, embedded with dental stone in a stainless ring.

For the strains calculated with the FE model, maximum and minimum principal strains at the nodes corresponding to the gauge-bonded locations on the tooth were determined. The strain values obtained from the FE model were compared with the strain values from the strain gauge technique using linear regression analysis to validate the FE model results. The independent variable was the strain in the FE model, and the dependent variable was the average strain measured with the strain gauge technique.

\section{RESULTS}

Figure 3 shows a multiplanar reconstruction image, CT slice images and semi-transparent reconstruction imaging of the tooth. In this study, $3372 \mathrm{D}$ grayscale images were obtained with an image resolution of 27 $\mu \mathrm{m}$ per pixel from the CT data. The slice images showed clearly distinct differences in grayscale for regions of enamel, dentin and pulp. The semitransparent colored image was acquired by volume rendering of three data set, which were extracted based on a certain range of grayscales. Pulp in the tooth tissues and CEJ on the tooth surface (white line in green region) were visible in the image, while a thinly overlapped cementum layer could be observed around the cervical root. Figure 4 shows the models generated from the CT data. The numbers of point clouds set for the tooth, dentin and pulp were 110,148, 92,007 and 22,160, respectively. For the final assembled solid model of the tooth, an FE mesh model comprising 20,773 elements and 30,718 nodes was generated.

Figure 5 shows vector plots of calculated 

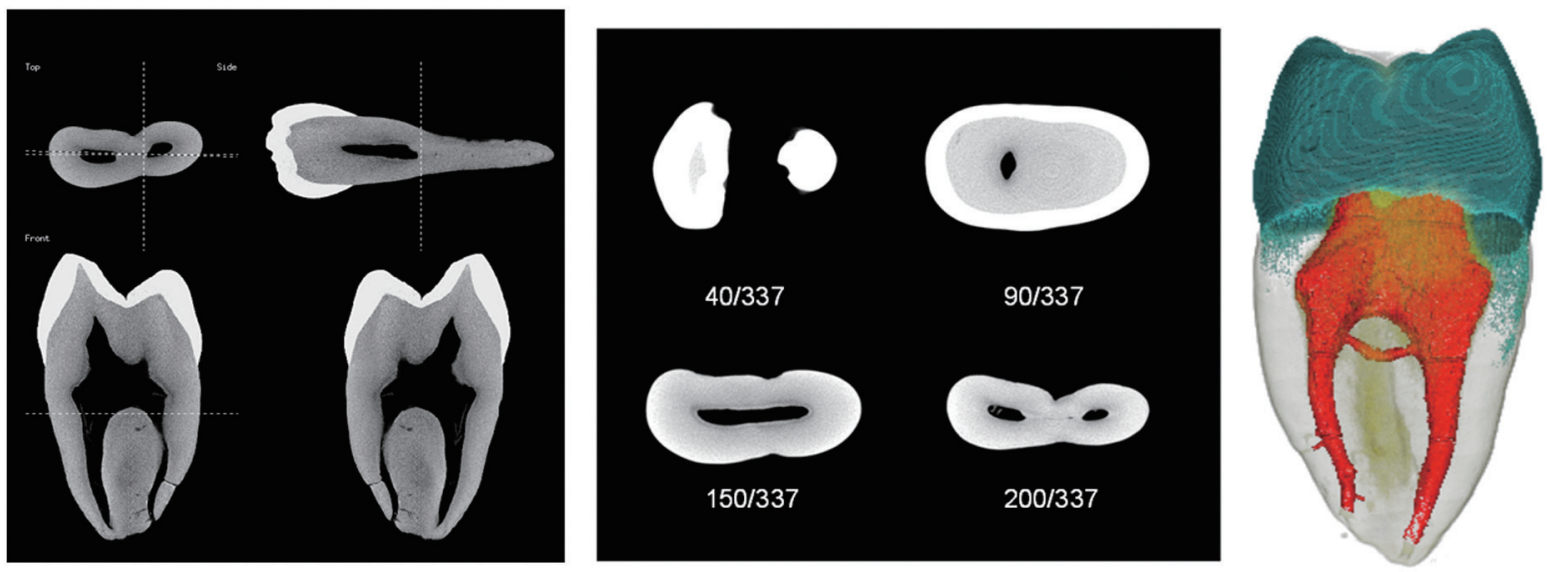

Fig. 3 CT images of the tooth.

Left: multiplanar reconstruction image. Center: representatives of CT slice images. Right: semi-transparent image. Pulp=red; dentin=white; enamel=green
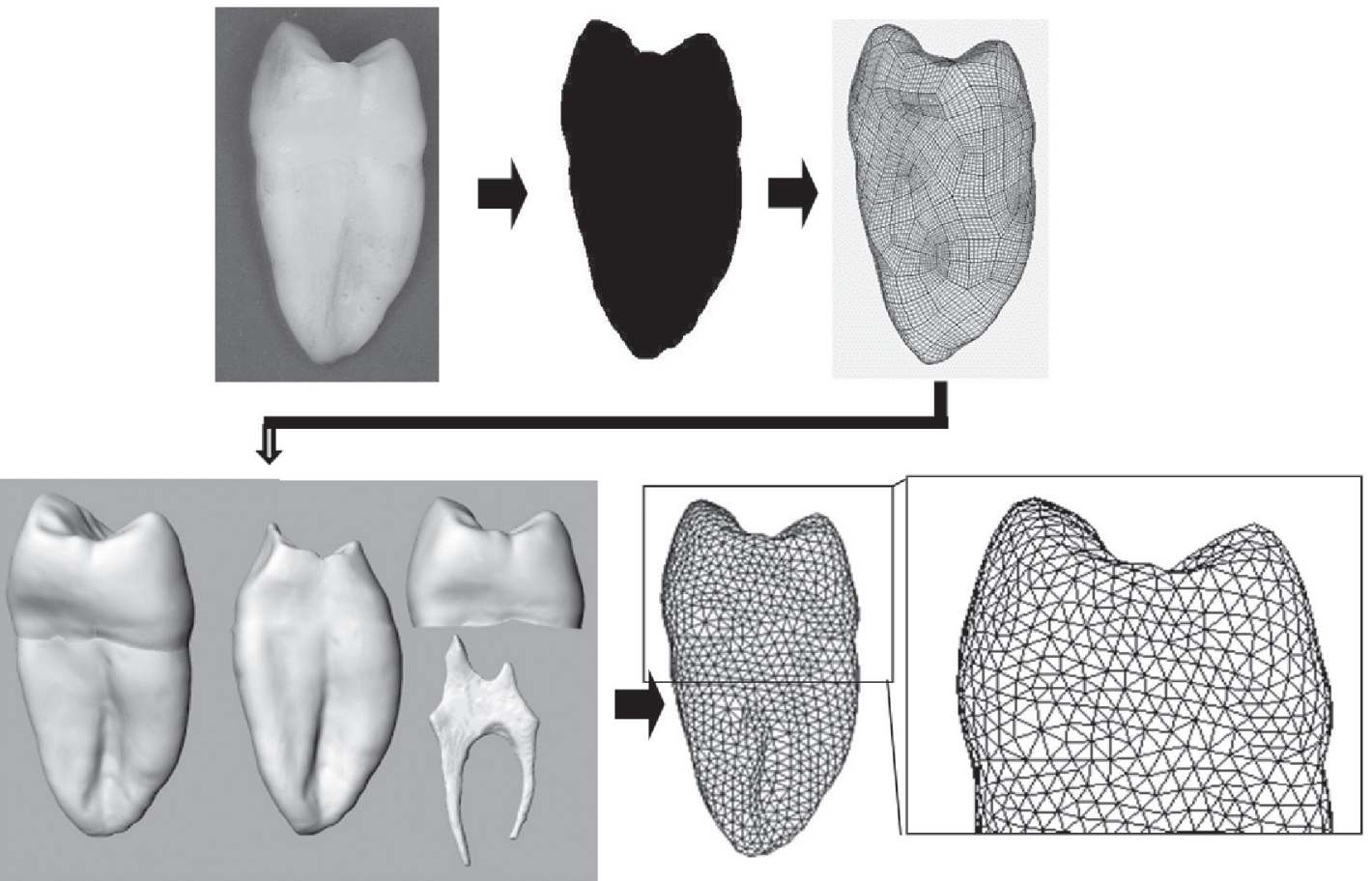

Fig. 4 Original tooth and models of each step.

Upper left original tooth. Upper center: point cloud of outer tooth. Upper right: surface model. Lower left: solid models. Lower center: FE mesh model. Lower right: close-up view of the mesh.

principal strain distributions in bucco-lingual cervical regions at $\mathrm{BO}, \mathrm{BC}$ and $\mathrm{BI}$ under loading. The strain data from the FE model and strain gauge technique are listed in Table 1. For the FE model, when a load was applied to the BO point, strains longitudinally estimated in the buccal and lingual cervical regions were maximum principle strain in tension and minimum principle strain in compression, respectively, and vice versa when the $\mathrm{BC}$ and $\mathrm{BI}$ points were subjected to loading. Similar behavior was also observed when a load was applied to the LO, LC and LI points for the FE model. The strains of the root cervical regions were larger than those of the coronal cervical regions. These strain behaviors estimated in the FE model were similarity observed in the measured strains.

Figure 6 shows the correlation between the strains calculated by the FE model and average 


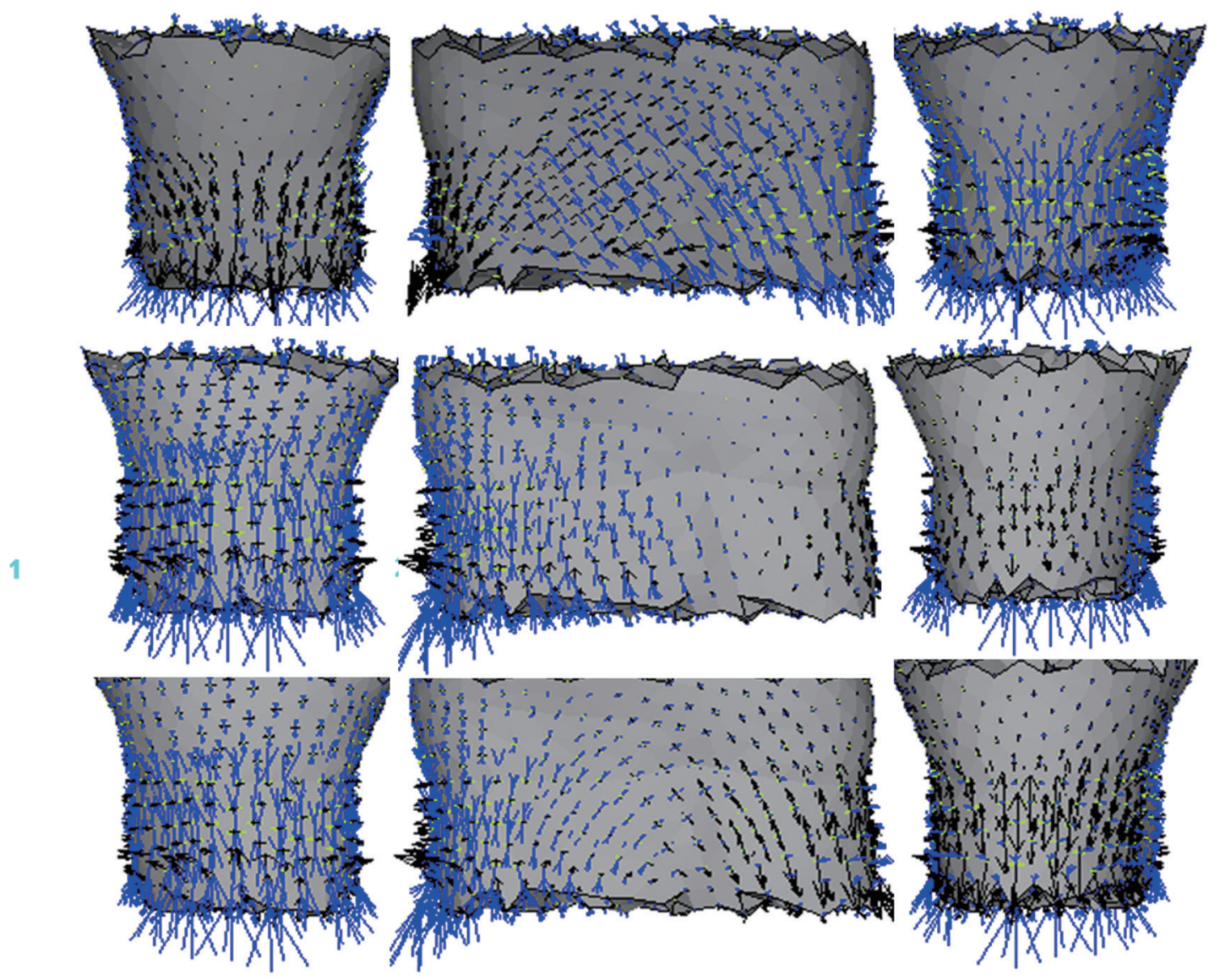

Fig. 5 Vector plots of calculated principal strain displacements at bucco-lingual cervical regions.

Black and blue vectors show maximum and minimum principal strains, respectively. Upper row: BO loading. Middle row: BC loading. Lower row: BI loading. Left column: buccal view. Center column: mesial view. Right column: lingual view.

Table 1 Measured (average and SD) and calculated strains at bucco-lingual cervical regions

$\left(10^{-6}\right)$

\begin{tabular}{|c|c|c|c|c|c|c|c|c|c|c|c|c|}
\hline & \multicolumn{6}{|c|}{ Buccal cervical region } & \multicolumn{6}{|c|}{ Lingual cervical region } \\
\hline & \multicolumn{3}{|c|}{ Crown } & \multicolumn{3}{|c|}{ Root } & \multicolumn{3}{|c|}{ Crown } & \multicolumn{3}{|c|}{ Root } \\
\hline & $\varepsilon(\mathrm{cal})$ & $\varepsilon(\mathrm{n}$ & nea) & $\varepsilon(\mathrm{cal})$ & $\varepsilon(\mathrm{n}$ & nea) & $\varepsilon(\mathrm{cal})$ & $\varepsilon(\mathrm{n}$ & ea) & $\varepsilon(\mathrm{cal})$ & $\varepsilon(\mathrm{n}$ & lea) \\
\hline $\mathrm{BO}$ & -4.2 & -52.1 & ( 48.8) & 187.4 & 127.8 & (135.8) & -20.7 & -17.5 & $(25.2)$ & -359.8 & -247.8 & (144.0) \\
\hline $\mathrm{BC}$ & -121.0 & -285.9 & $(107.9)$ & -449.9 & -457.4 & (184.3) & 26.8 & 59.8 & $(28.5)$ & 119.0 & 255.9 & (196.1) \\
\hline BI & -194.8 & -351.1 & $(273.2)$ & -881.7 & -708.7 & $(325.5)$ & 65.9 & 68.6 & $(44.1)$ & 563.7 & 353.8 & $(170.7)$ \\
\hline LI & 49.7 & 122.3 & $(29.4)$ & 484.3 & 451.9 & (101.8) & -141.5 & -202.5 & $(57.9)$ & -715.2 & -431.5 & $(306.4)$ \\
\hline $\mathrm{LC}$ & 27.7 & 71.6 & ( 19.3) & 111.6 & 224.4 & $(91.0)$ & -104.1 & -190.3 & $(82.1)$ & -368.8 & -389.3 & $(211.4)$ \\
\hline $\mathrm{LO}$ & -11.3 & -22.7 & $(24.7)$ & -329.6 & -211.9 & (115.9) & -4.3 & -69.5 & $(58.7)$ & -72.9 & -57.1 & (376.9) \\
\hline
\end{tabular}




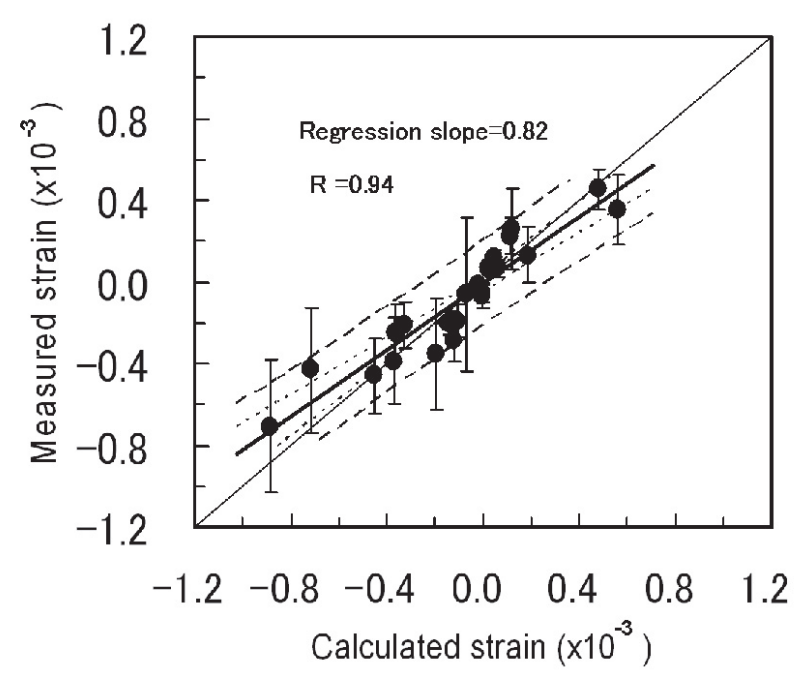

Fig. 6 Correlation between the strains calculated by the FE model and average strains measured by the strain gauge method $(n=5)$.

Solid line shows the regression line. Short and long dashed lines show the 95\% confidence interval for the regression and population, respectively. Vertical lines show the SD of measured strains $(n=5)$.

strains measured by the strain gauge method. The result of the linear regression analysis showed that there is a significantly strong positive correlation between the calculated and measured strain $(r=0.94$, $\mathrm{p}<0.001$ ) with a standard error of 0.0001 . The regression slope and its standard error are 0.82 and 0.06 , respectively, while the regression slope with a $95 \%$ confidence interval of 0.70 to 0.94 is significantly different from 1 (Y=X).

\section{DISCUSSION}

The results of $\mathrm{FE}$ analysis require experimental validation, due to the effects of the $\mathrm{FE}$ model assumputions ${ }^{16,17)}$. In the field of tooth biomechanics, however, there are few reports on the validation of $\mathrm{FE}$ models of the teeth ${ }^{18-25)}$. Reeh et al. have validated tooth $\mathrm{FE}$ models with the strain gauge technique, although for $2 \mathrm{D}$ models ${ }^{19)}$. Parmard et $a l .{ }^{21,22)}$ and Lee et $a l .{ }^{23)}$ have validated 3D FE models reconstructed using cross-sectional histological images. However, these reports have not clearly demonstrated the statistical correlation between the results with the $\mathrm{FE}$ models and experimental methods. In this study, according to the high correlation coefficient (0.94) and the low standard error (0.06) of the regression analysis, the FE model reconstructed from the $\mathrm{CT}$ image shows qualitatively good agreement with the result of the experimental model. This indicates that the FE model can be considered valid with acceptable accuracy for use as a simulation model of the actual strain in the tooth.

In this study, the FE model constructed from CT showed a tendency to quantitatively underestimate the strains as compared with the experimentally measured ones, as indicated by the value of the regression slope in Fig. 6, which was less than 1. Some of the causes for this underestimation of the strain magnitudes may be due to both the uncertainty in correlating experimental conditions and that in the FE model. Obviously from the scattering in the measured strains shown in Table 1, the experimental method has unavoidable uncertainties in both geometry ${ }^{29)}$ and material properties ${ }^{28,30)}$ of the tooth, and experimental conditions such as strain gauge position and loading position. The strain gauge technique, therefore, would present practical challenges in that it is difficult to adjust the gauges, possibly resulting in the unavoidable scattering of the results.

On the other hand, the accuracy of the results of the FE model depends on the accuracy of input parameters; the error depends on geometry, materials properties, boundary conditions, and loads applied. The FE analysis is a deterministic approach in that the output is determined once the set of selective input parameters and the geometry of the model have been specified. In this study, one geometric model, reconstructed from CT data for a specific tooth and the average elastic modulus of enamel and dentin in the literature, was selected. Furthermore, we made the assumptions that both dentin and enamel were homogeneous, isotropic and elastic despite full representation of the tooth tissues in order to simplify the calculations for the model. Such a deterministic and assumptive approach in constructing FE models may be attributed to the inaccuracy of the FE results. Therefore, a statistical approach incorporating these uncertainties in $\mathrm{FE}$ analysis of biological tooth tissues is important to provide a more accurate evaluation. Furthermore, the accuracy of $\mathrm{FE}$ results is also dependent on element and node sizes of the FE model. The element size of the FE model in this study was finer than compared with the FE models previously reported ${ }^{22,23)}$. The FE analysis program used in this study has an allowable limit of 32,000 nodes. It appears, therefore, that the size limit had been generated in the FE mesh model, which could be reconstructed with acceptable accuracy. The effect of element size on accuracy of the FE model requires further investigation.

This study shows that an FE model of a tooth can be reconstructed through geometry-based modeling, in which a combination of several commercially available computer-aided techniques are used to extract geometrical information from $\mathrm{CT}$ 
data and to generate the model. Previously, several successful works on FE modeling of teeth from CT data have applied some efficient geometry extractionCAD modeling combinations ${ }^{11-13,16)}$. Such combinations might result in a considerable reduction in the time consumed in creation of FE models, as compared with traditional methods of modeling from CT data. In this study, however, manual operations in both $\mathrm{CAD}$ and $\mathrm{FE}$ mesh modeling were very timeconsuming and required both considerable experience and skill, although segmentation and surface modeling were mostly automated. Therefore, fully automated and more efficient modeling techniques using CT data of the teeth would facilitate creation of FE models without considerable skill.

In conclusion, a 3D FE tooth model could be constructed using micro-CT data and a combination of several commercially available application techniques with acceptable accuracy, although the FE modeling process starting from CT data was not fully automated. The FE model presented here provides a useful means to three-dimensionally explore the biomechanical characteristics of tooth abfraction lesions under occlusal loading.

\section{ACKNOWLEDGEMENTS}

We express our sincere thanks to Mrs. Hirohide Kaida and Masaaki Koganemaru of Fukuoka Industrial Technology Center for supplying the CT system used in this study and their important contributions to the experiments. This work was supported in part by the Grant-in-Aid for Scientific Research (C) 18592095 from the Ministry of Education, Culture, Sports, Science and Technology of Japan, 2006.

\section{REFERENCES}

1) Arturo NN. Dental biomechanics, 1st ed, Taylor \& Francis, New York, 2004, pp. 211-238.

2) Jaroslav M. Finite element modelling and simulations in dentistry: A bibliography 1990-2003. Comput Methods Biomech Biomed Engin 2004; 7: 277-303.

3) Nagasawa S. The principle and some applications in dentistry of the finite-element method. J Matsumoto Dent Coll 2007; 33: 10-20.

4) Andréa BM, Luiz CP, Andréia RCCDC. Finite element analysis in2D and 3D models for sound and restored teeth. Proceedings of ABAQUS users' conference; 2006 May 329-343; Boston, USA; 2002. pp. 329-343.

5) Litonjua LA, Andreana S, Patra AK, Cohen RE. An assessment of stress analyses in the theory of abfraction. Biomed Mater Eng 2004; 14: 311-321.

6) Romeed SA, Fok SL, Wilson NHF. A comparison of $2 \mathrm{D}$ and $3 \mathrm{D}$ finite element analysis of a restored tooth. J Oral Rehabil 2006; 33:209-215.
7) Wei L, Michael VS, Qing L, Grant PS. Towards automated 3D finite element modeling of direct fiber reinforced composite dental bridge. J Biomed Mater Res B Appl Biomater 2005; 74: 520-528.

8) Rahimi A, Keilig L, Bendels G, Klein R, Buzug TM, Abdelgader I, Abboud M, Bourauel C. 3D Reconstruction of dental specimens from 2D histological images and CT-Scans. Comput Methods Biomech Biomed Engin 2005; 8: 167-176.

9) Verdonschot N, Fennis WM, Kuijs RH, Stolk J, Kreulen CM, Creugers NH. Generation of 3D finite element models of restored human teeth using micro-CT techniques. Int J Prosthodont 2001; 14: 310-315.

10) Lin $\mathrm{CL}$, Lee HE, Wang $\mathrm{CH}$, Chang $\mathrm{KH}$. Integration of CT, CAD system and finite element method to investigate interfacial stresses of resin-bonded prosthesis. Comput Methods Programs Biomed 2003; 72: 55-64.

11) Clement R, Schneider J, Brambs HJ, Wunderlich A, Geiger M, Sander FG. Quasi-automatic 3D finite element model generation for individual singlerooted teeth and periodontal ligament. Comput Methods Programs Biomed 2004; 73: 135-144.

12) Gomes de Oliveira S, Seraidarian PI, Landre J Jr, Oliveira DD, Cavalcanti BN. Tooth displacement due to occlusal contacts: a three-dimensional finite element study. J Oral Rehabil2006; 33: 874-880.

13) Magne P. Efficient $3 \mathrm{D}$ finite element analysis of dental restorative procedures using micro-CT data. Dent Mater 2007; 23: 539-548.

14) Ichim I, Schmidlin PR, Kieser JA, Swain MV. Mechanical evaluation of cervical glass-ionomer restorations: 3D finite element study. J Dent 2007; 35: 28-35.

15) Ichim I, Li Q, Loughran J, Swain MV, Kieser J. Restoration of non-carious cervical lesions Part I. Modelling of restorative fracture. Dent Mater 2007; 23: 1553-1561.

16) Nakajima A, Murata M, Tanaka E, Arai Y, Fukase Y, Nishi Y, Sameshima G, Shimizu N. Development of three-dimensional FE modeling system from the limited cone beam CT images for orthodontic tipping tooth movement. Dent Mater J 2007; 26: 882-891.

17) Huysmans MC, Van der Varst PG. Finite element analysis of quasistatic and fatigue failure of post and cores. J Dent 1993; 21(1): 57-64.

18) Magne P, Tan DT. Incisor compliance following operative procedures: a rapid $3 \mathrm{D}$ finite element analysis using micro-CT data. J Adhes Dent 2008; 10: $49-56$.

19) Reeh ES, Ross GK. Tooth stiffness with composite veneers: a strain gauge and finite element evaluation. Dent Mater 1994; 10: 247-252.

20) Asundi A, Kishen A. A strain gauge and photoelastic analysis of in vivo strain and in vitro stress distribution in human dental supporting structures. Arch Oral Biol 2000; 45: 543-50.

21) Palamara D, Palamara JE, Tyas MJ, Messer HH. Strain patterns in cervical enamel of teeth subjected to occlusal loading. Dent Mater 2000; 16: 412-419.

22) Palamara JE, Palamara D, Messer HH. Strains in the marginal ridge during occlusal loading. Aust Dent J 2002; 47: 218-222. 
23) Lee HE, Lin CL, Wang $\mathrm{CH}$, Cheng $\mathrm{CH}$, Chang $\mathrm{CH}$. Stresses at the cervical lesion of maxillary premolar--a finite element investigation. J Dent 2002; 30: 283290.

24) Lertchirakarn V, Palamara JE, Messer HH. Finite element analysis and strain-gauge studies of vertical root fracture. J Endod 2003; 29: 529-534.

25) Soares PV, Santos-Filho PC, Gomide HA, Araujo CA, Martins LR, Soares CJ. Influence of restorative technique on the biomechanical behavior of endodontically treated maxillary premolars. Part II: strain measurement and stress distribution. J Prosthet Dent 2008; 99: 114-122.
26) Robert McNeel \& Associates. Rhinoceros User's Guide 2002; Ver 3.0: 146-149.

27) Chen KK, Miyake K, Shono Y, Terashita M. Japan J Consev Dent 2000; 43: 870-876.

28) Sakamoto M, Sato K, Koda F, Sakai J, Kobayashi K, Tanabe Y, Itoh H. Measurements of mechanical properties of hard tissues using nanoindentation tests. Proceedings of JSEM. 2003; 3: 127-130.

29) Shillingburg HT Jr, Kaplan MJ, Grace SC. Tooth dimensions-a comparative study. J South Calif Dent Assoc 1972; 40: 830-839.

30) Sakamoto M. Biomechanics of hard tooth tissues. Journal of JSNDI 2005; 54:284-288. 